\title{
La répression de l'infraction de viol d'enfant dans les zones rurales en République Démocratique du Congo
}

\author{
Par : KITWA KALENGA Matthieu, Assistant à l'Université de Likasi
}

\section{INTRODUCTION}

\section{PRESENTATION DU SUJET}

D'aucun n'ignore que les violences sexuelles faites aux femmes et surtout les viols d'enfants sont devenus monnaie courante en République Démocratique du Congo rendant ainsi la condition de l'enfant très vulnérable qu'elle ne l'était déjà par rapport à son milieu, son manque de maturité physique et émotionnelle nécessitant une attention particulière pour sa protection.

La constitution de la République Démocratique du Congo du 18 février 2006 en son article 123, point 16, consacre une place importante aux personnes vulnérables, notamment aux enfants entant que renouvellement de l'être et de la vie, en s'engageant dans la voie de faire de leur protection son cheval de bataille.

Bien que la République Démocratique du Congo dispose des moyens de persuasion pour décourager les délinquants des violences sexuelles, ceux-ci ne désarment pas pour autant sur tout dans les zones rurales où le taux de ces infractions reste élevé.

Cette situation s'explique par le fait que dans ces milieux, les citoyens ignorent non pas seulement leurs droits mais aussi, ils ne sont pas informés ; les organes de répression font dans la plupart de cas défaut et lorsqu'ils sont là, il faut effectuer une longue distance pour les atteindre. Surtout, certaines coutumes et traditions ne favorisent pas l'épanouissement des enfants notamment des filles.

En dépit du fait que le viol soit une infraction qui se commette en cachette et que sa preuve soit très difficile à apporter, souvent quand les victimes se proposent de porter l'affaire en justice, généralement elles le font en retard pendant que les preuves deviennent difficiles à reconstituer ou à apporter. Aussi, les facteurs psychologiques notamment le traumatisme psychosociale tels que le viol, l'inceste et la molestation sexuelle ont un impacte négatif sur la vie de la victime. L'article 171 de la loi $n^{\circ}$ 09/001 du 10 janvier '2009 portant protection de l'enfant répute le viol avec une personne âgée de moins de dix-huit ans, viol à l'aide des violences. Il en va sans dire que tout rapport sexuel avec des personnes âgées de moins de dix-huit ans, avec ou sans consentement de celles-ci est réputé viol avec violences.

Pour asseoir la conviction du juge afin d'arracher la condamnation de l'auteur de l'infraction, les parties au procès notamment la partie victime doit prouver la véracité des faits allégués.il incombera à la partie victime d'apporter d'abord par le moyen de droit la preuve de sa minorité, puis les faits même se rapportant au viol. Dans cette démarche, les actes dressés tant par l'officier de l'état civil, par le médecin que par le psychologue s'avèrent indispensables pour établir la véracité des faits allégués. Il sera question pour nous dans le cadre de cette réflexion de démontrer comment les actes de l'état civil et ceux établis par le médecin ainsi que le psychologue sont indispensables pour la répression de viol d'enfant commis dans les zones rurales en République Démocratique du Congo ainsi que pour la prise en charge efficace des victimes.

\section{Chapitre I. DEFINITION DES CONCEPTS DE BASE}

Il nous sera difficile d'aborder notre thème sans avoir au préalable définir certains concepts clés

A. VIOL :

Plusieurs doctrinaires définissent le viol, mais dans le cadre du présent travail le viol est défini comme, le rapport sexuel avec une personne qui a un bas âge de consentement. Le rapport sexuel dans telles conditions est considéré comme un viol légal même si la victime coopère. ${ }^{1}$

Jadis, le viol était considéré comme une conjonction sexuelle obtenue contre le gré de la femme. Ce qui n'est pas le cas aujourd'hui dans la mesure où le concept viol revêt plusieurs autres formes qui sont :

- L'intromission d'un objet autre que le sexe de l'homme dans le vagin de la femme ;

- L'intromission du pénis dans un orifice autre que le vagin de la femme ;

\footnotetext{
${ }^{1}$ RADJA BIN SAIDI.P, Introduction à la psychologie de l'adulte, ed. Fondation. Dr Pierre, 2015, Lubumbashi 2015, p128

DOI: $10.9790 / 0837-2206023543 \quad$ www.iosrjournals.org $35 \mid$ Page
}


-L'introduction d'un objet quelconque dans les orifices du corps d'autrui n'ayant pas vocation $\mathrm{fr} r$ sexuelle intrinsèque et/ou l'utilisation de ces orifices dans un but sexuel ;

-L'exposition du corps de l'enfant par la femme à des attouchements par une partie du corps ou par un objet quelconque.

Il ressort de ces éléments que le viol entant que manifestation de l'agression sexuelle englobant en son sein des faits qui parfois sont loin de réaliser un simple contact physique, peut être défini comme une invasion physique de nature sexuelle commise sur la personne d'autrui sous l'emprise de la contrainte.

\section{B. ENFANT}

Dans le cadre du présent travail, Il est à noter qu'il est difficile de parler de l'enfant ou de l'adulte sans se référer à l'âge.

En asseyant de définir le terme âge, les chercheurs dans ce domaine ont trouvé qu'il y a quatre sortes de groupes d'âges à savoir, l'âge chronologique, social, psychologique et biologique.

- Age chronologique : se mesure à partir de la naissance d'une personne dans un contexte social bien déterminé. Dans la culture moderne, le passage de 365 jours «équivaut à un an.

- Age social : Est un système qui sépare les gens selon leurs différents niveaux de maturité d'après les fonctions sociaux. L'âge social est marqué par les événements de la vie tels que le mariage, la naissance du premier enfant, ...

- Age psychologique : Est un système par lequel la maturité est mesurée en termes de capacités de s'adopter et de connaitre.

- Age biologique : Se définit en termes d'une maturité biologique provoquée par l'hérédité et l'environnement. Et il est possible qu'une personne soit biologiquement vielle mais chronologiquement jeune. ${ }^{2}$

Dans le cas de la presente réflexion, nous nous sommes beaucoup plus intéressés à l'âge chronologique dans le mesure où c'est la conception qui influence les scientifiques et constitue le facteur déterminant du développement humain.

Les taches spécifiques de l'individu peuvent être regroupées en cinq stades à savoir : la première enfance, l'enfance moyenne, l'adolescence, la première vue adulte et l'âge moyen.

Les études scientifiques démontrent que de 0 à18, la personne est en pleine métamorphose, c'est l'âge d'apprentissage. Et la vie adulte ne commence qu'à partir de 18 ans.

Pour Coleman, de 12 à 18 ans la personne doit:

- Développer clairement son identité et sa confiance en soi ;

- S'adopter aux changements du corps ;

- Etablir une indépendance émotionnelle vis-à-vis des parents ;

- Développer des relations plus mures avec les paires d'âge ;

- Sélectionner et séparer à une profession ;

- Apprendre à se tourner vers les autres

- Etablir des valeurs mures et une responsabilité sociale ;

- Se préparer au mariage et à la vie familiale ${ }^{3}$.

La définition de l'enfant correspond à celle donnée par les instruments juridiques internationaux du droit des enfants qui tiennent compte de l'âge chronologique.

La convention relative aux droits de l'enfant stipule en son article 1 que « Un enfant s'entend de tout être humain âgé de moins de moins dix-huit ans», à l'instar de l'article 2 de la charte Africaine sur les droits et le bien-être de l'enfant et de l'article 2.1 de la loi nº9/001 du 10 janvier 2009 portant protection de l'enfant.

De ce qui précède l'enfant est donc toute personne âgée de moins de 18 ans.

\section{ZONE RURALE}

Le concept zone rurale veut dire : l'espace constitué de la population, des Territoires et des ressources des campagnes. Environs deux tiers de la population de la République Démocratique du Congo vit dans les zones rurales. La population qui vit dans les zones rurales est confrontée à beaucoup de difficultés qui sont liées notamment au manque d'infrastructures pouvant abriter les différents services de l'Etat et à l'absence du personnel qualifié dans plusieurs domaines de la vie (Justice, Santé, social...)

${ }^{2}$ RADJA SAIDI.P. Opcit, page 26

${ }^{3}$ COLEMAN.J, identity and life cycle, cité par RADJA SAIDI.P, Opcit, page 26

DOI: 10.9790/0837-2206023543 www.iosrjournals.org $36 \mid$ Page




\section{Chapitre II. L'INFRACTION DE VIOL D'ENFANT.}

Dans cette partie consacrée à l'infraction de viol d'enfant, il sera question d'analyser les éléments constitutifs de cette prévention et de parler de l'administration de la preuve, des organes poursuivants ainsi que du tribunal compétent. Dans la plus part de cas criminels, il est indispensable de savoir les facteurs responsables de la criminalité et sur lesquels il faudrait agir en priorité. Les causes de la délinquance sont multiples, mais selon Jean Pradel, il identifie quelques-unes notamment la pauvreté, le taux de chômage, l'influence du milieu, la consommation de la drogue, l'handicape physique ou mental, l'absence de célérité du système judiciaire et servitude de la peine, l'isolement social et la perte de l'esprit communautaire ${ }^{4}$.

De ce fait ces causes engendrent des conséquences fâcheuses mettant en péril l'idéal social. Parmi ces conséquences on peut noter d'abord ; les conséquences subies par la victime (blessures physiques, traumatismes psychologique, modification du style de vie, peur du crime, etc... en suite, celles touchant le contrevenant (détérioration des relations familiales et sociales); et fondement, celles modifiant la société (alternation de la qualité de vie, sentiment croissant d'incertitude chez les citoyens, agressivité collective à la hausse).

Cependant, la connaissance des causes et des conséquences de ce phénomène est nécessaire mais insuffisante pour extirper le mal. Il faut donc des actions efficaces et concertantes de tous les responsables à savoir l'état, société civile, enseignants, familles et certains psychologues en vue de la réintégration mentale. Car l'absence d'un élément indispensable à l'organisme crée des perturbations psychiques déclenchées chez un sujet par un choc émotionnel ${ }^{5}$.L'Etat congolais de par les instruments et institutions juridiques, protège ses citoyens contres toutes les atteintes dirigées contre eux ainsi que contre leurs droits individuels.

Le viol d'enfant porte atteinte à la vie des personnes et constitue une expérience traumatisante tant sur le plan émotionnel que physique ; mais pour être réprimée, les éléments constitutifs que voici doivent être réunis.

\section{A. ELEMENTS MATERIELS}

1) CONDITION PREALABLE (la minorité d'âge de la victime)

Au regard de l'art.2.1 de la loi précitée est considéré comme enfant, toute personne âgée de moins de 18 ans. Et pour qu'il y ait viol, la victime doit être préalablement âgée de moins de 18 ans.

\section{2] LES ACTES MATERIELS DE VIOL}

Le législateur congolais a prévu plusieurs modes de commission de viol. Et dans le cadre du présent travail nous allons tenter de les analyser tous. Notons cependant, que ces modes de commission de viol ne sont pas cumulatifs, c'est-à-dire, un seul mode suffit pour le viol soit établi.

\section{CONJONCTION SEXUELLE}

Pour que cette infraction de viol soit établie, la loi exige qu'il y ait conjonction sexuelle, c'est-à-dire, pénétration de l'organe de l'homme dans le vagin de la femme. Il y a conjonction sexuelle peu importe qu'il y ait eu atteinte de l'orgasme ou non, que l'agresseur sexuel ait été satisfait ou pas, qu'il ait causé des lésions corporelles à la victime ou pas. Nous estimons qu'à défaut de d'administrer la preuve de la conjonction sexuelle, à défaut d'expertise médicale, la seule déclaration de la victime ne saurait suffire pour confirmer l'existence des rapports sexuels. La conjonction sexuelle peut être soit l'œuvre de l'homme comme c'est souvent les cas, soit de la femme. Il y a viol lorsque l'homme introduit complètement ou superficiellement son pénis dans le vagin d'une enfant. Il y a également viol lorsqu'une femme oblige un enfant, à introduire même superficiellement son organe sexuel dans son vagin. Peu importe le moyen utilisé.

Il ressort de ce qui vient d'être dit que la victime du viol peut être aussi bien du sexe masculin que féminin. Contrairement à l'ancienne législation qui prévoyait que pour qu'il y ait viol il fallait nécessairement qu'il y ait intromission de la verge de l'auteur dans le vagin de la femme, la loi n 09/001 du 10 janvier 2009 portant protection de l'enfant ne fait pas de la pénétration du pénis dans le vagin de la femme une condition indispensable, car même sans pénétration du pénis dans le vagin, le viol peut se commettre.

\section{- L'intromission d'un organe sexuel dans l'anus, dans la bouche ou dans tout autre orifice du corps}

Le législateur congolais retient le viol lorsqu'il y a intromission même superficielle de l'organe sexuel dans l'anus, dans la bouche ou dans tout autre orifice du corps d'une femme ou d'un homme par un organe sexuel, par toute autre partie du corps ou par un objet quelconque. C'est le cas par exemple de l'intromission du pénis dans la bouche ou dans l'anus d'un enfant.

\section{- d'une autre partie du corps ou d'un objet dans le vagin.}

\footnotetext{
4 J.PRADEL, évolution Criminelle, 3eme édition, PUF, Paris, 1956, p30

${ }^{5}$ J.FERRIE, la psychologie judiciaire, une perspective européenne, $1^{\text {er }}$ éd SBN, Bruxelles, P,2003.

DOI: 10.9790/0837-2206023543 www.iosrjournals.org $37 \mid$ Page
}


Toute personne qui aura introduit, même superficiellement, toute autre partie du corps ou un objet quelconque dans le vagin de la victime, commet le viol. C'est le cas par exemple de l'agresseur qui introduit dans le vagin de la victime soit sa langue, ses ongles, ses doigts soit un bâton...

\section{- L'exposition de l'organe sexuel à des attouchements.}

Commet également le viol toute femme qui oblige un enfant à exposer son organe sexuel à des attouchements par une partie de son corps ou par un objet quelconque. C'est le cas d'une femme qui, pour exciter un enfant, se met à caresser son pénis. Par attouchement, il faut entendre : caresses sexuelles sans consentement de la victime, ou action de toucher légèrement avec la main. Le viol ici concerne les caresses sexuelles qui ne sont pas des actes de pénétration mais qui portent atteinte à la vie de l'enfant.

Il est à noter que ce genre de viol passe inaperçu à cause de la preuve des faits qui est parfois difficile à démontrer.

\section{-L'obligation d'une personne à pénétrer l'anus, la bouche ou tout autre orifice du corps par un organe sexuel, par toute autre partie du corps ou par un objet quelconque.}

Toute personne qui oblige un homme, dans le cas sous examen, un enfant à pénétrer même superficiellement son anus, sa bouche, ou tout autre orifice de son corps par un organe sexuel, par toute autre partie du corps ou par un objet quelconque, commet le viol. C'est l'exemple d'une femme qui, profitant de la naïveté d'un enfant, l'oblige à introduire son organe sexuel dans le sien.

\section{2) Absence de consentement de la victime.}

L'on entend par consentement, un accord entre personnes, manifesté par l'expression de l'acceptation de chacune de parties. Et son absence sous entend le défaut de consentement. Il est de la volonté de la loi que pour que l'infraction de viol d'enfant soit établie, il faut qu'il y ait absence de consentement. La victime ne peut donc pas être consentante de l'acte sexuel. Pour que le viol soit établi, la volonté de la victime à consentir doit être paralysée par des violences ou menaces graves, par contrainte à l'endroit de la victime, directement ou par l'intermédiaire d'un tiers, soit à l'occasion d'un environnement coercitif, soit en abusant de la victime qui, par le fait d'une maladie, par l'altération de ses facultés ou par toute autre cause accidentelle aurait perdu l'usage de ses sens ou en aurait été privé. Dans la mesure où les mineurs sont incapables de par la volonté de loi, il y a toujours présomption d'absence de consentement de la victime. Lorsque la victime du viol est un enfant, la présomption du défaut de consentement est donc irréfragable. C'est dans cette logique que l'auteur de l'infraction de viol d'enfant ne saura pas se disculpé sous prétexte qu'il ignorait l'âge de la victime.

Il est à noter que l'élément déclencheur de l'infraction de viol d'enfant est l'âge de la victime. Qu'elle soit consentante ou pas, qu'il y ait violence ou ruse, des lors que la victime a moins de dix-huit ans, il y a viol et le défaut de consentement est acquis. Il est donc conseiller aux coureurs des jupons de bonne fois si ils existent, de toujours vérifier l'âge de leurs partenaires sinon, ils n'échapperont pas en cas des poursuites et n'auront d'autres excuses que de subir la rigueur de la loi. La loi est dure mais c'est la loi, dit-on !

\section{B. ELEMENT INTELLECTUEL}

Le viol d'enfant étant une infraction intentionnelle, la seule volonté de l'agresseur d'imposer, de consommer des relations sexuelles ou de pénétrer par un organe sexuel le vagin, l'anus ou tout autre orifice de la victime par un objet ou toute autre partie du corps, à l'aide de violences ou menaces graves, ou par contrainte, par surprise, par pression psychologique, soit à l'occasion d'un environnement coercitif, soit en abusant d'une personne malade, altérée mentale ou victime de toute autre cause accidentelle ou privée de ses sens par quelques artifices, suffit pour que l'élément moral soit constitué. Il ne fait l'ombre d'aucun doute que la seule volonté de l'agresseur de satisfaire ses besoins sexuels sur une personne âgée de moins de dix-huit ans, quels que soient les moyens utilisés, que la victime soit consentante ou pas, suffit pour qu'il y ait viol d'enfant.

\section{* L'ADMINISTRATION DE LA PREUVE EN CAS DE VIOL D'ENFANT}

Dans cette partie relative à l'administration de la preuve en cas de viol d'enfant, il sera question pour nous de parler des institutions qui interviennent dans le déclenchement des poursuites, de la juridiction compétente pour connaitre de l'affaire de viol d'enfant, ainsi que de la preuve en cette matière.

\section{Des poursuites}

Dans la phase dite préjuridictionnelle, deux institutions interviennent pour la recherche des infractions, à savoir l'officier de police judiciaire qui, dans la plupart de cas est à proximité des justiciables entant que première autorité à entrer en contact avec le délinquant et l'Officier du Ministère Public qui instruit et juge de l'opportunité des poursuites. 


\subsection{L'officier de police judiciaire}

Les officiers de police judiciaire constatent les infractions qu'ils ont mission de rechercher ; ils reçoivent des dénonciations, plaintes et rapports à ces infractions.

Ils consignent dans leurs procès-verbaux, la nature et les circonstances de ces infractions, le temps et les lieux où elles ont été commises, la preuve ou indices à charge de ceux qui en sont les auteurs présumés ainsi que les dépositions des personnes qui auraient été présentes ou auraient des renseignements à fournir. Ils interrogent les auteurs présumés des infractions et récupèrent leurs explications. Les procès-verbaux se terminent par le serment écrit : «Je jure que le présente procès - verbal est sincère ». Ils sont transmis directement à l'autorité compétente ${ }^{6}$.En matière de l'infraction de viol d'enfant, toute personne peut dès qu'il en a l'information, porter plainte et dénoncer le viol. C'est ainsi qu'hormis, le cas de la saisine d'office, les officiers de police judiciaire peuvent être saisis par voie de dénonciation ou de plainte. Dans le souci de rendre plus efficace et d'assurer une meilleure protection des mineurs victimes de viol, le législateur congolais a rendu obligatoire la dénonciation par toute personne, de toute forme de violence physique ou morale infligée a l'enfant ainsi que toutes menaces a sa santé et a son développement dont elle a connaissance. Ainsi, le nom dénonciation des violences commises sur un enfant est punie d'une amande de cent mille a deux cent mille francs congolais ${ }^{7}$.Qu'il soit saisi d'office ou par plainte, l'Officier de Police Judiciaire doit comprendre qu'en matière des violences sexuelles, notamment de viol d'enfant, son enquête est de portée immédiate. Elle est menée sans désemparer de manière à fournir à l'Officier du Ministère Public les principaux éléments d'appréciation. C'est pourquoi, l'Officier de Police Judiciaire saisi d'une infraction relative aux violences sexuelles en avise dans les 24heures l'Officier du Ministère Public dont il relève. Il est à signaler un comportement déplorable qui a élu domicile dans le chef des Officiers Police Judiciaire, sur tout de ceux évoluant dans les milieux ruraux, qui ne transmettent les dossiers à l'Officier du Ministère Public que lorsqu'ils se rendent compte que les parties ne sont pas à même de transiger faute des moyens oubliant qu'en matière de violences sexuelles l'amende transactionnelle ne s'applique pas.

\subsection{L'OFFICIER DU MINISTERE PUBLIC}

Les Officiers du Ministère Public peuvent exercer eux-mêmes toutes attributions des Officier de Police Judiciaire. Le Ministère Public recherche les infractions aux actes législatifs et réglementaires qui sont commises sur le territoire de la République ${ }^{8}$.Il ressort de ce qui précède, que les attributions du Ministère Public consistent à constater les infractions, à recevoir les dénonciations et plaintes, à consigner dans des procèsverbaux la nature et les circonstances de ces infractions, le temps et le lieu où elles ont été commises tout en prélevant les preuves ou indices à charge de ceux qui en sont les auteurs présumés ainsi que des personnes qui auraient été présentes, ou auraient des informations à fournir, à interroger les auteurs des infractions et à recueillir leurs explications. Bien plus, le Ministère Public saisit les cours et tribunaux.

L'Officier du Ministère Public doit comprendre que la procédure en matière de violences sexuelles requiert célérité et que l'enquête préliminaires en cette matière doit se faire dans un délai d'un moins maximum à partir de la saisine de l'autorité judiciaire.

Notons que le temps d'un mois imparti au Ministère Public pour mener les enquêtes préliminaires en matière de violence sexuelles n'est pas sans critiques dans la mesure où il n'est pas adapté à la réalité sur terrain. Certains Officiers de Police Judiciaire qui constatent en premier ces genres d'infraction sont très loin de l'Officier du Ministère Public et qu'il leur faut parcourir une très bonne distance pour atteindre celui-ci. Ceci est pareil tant pour les victimes qui voudraient porter leurs affaires à l'Officier Ministère Public que pour celui qui voudrait descendre sur terrain récolter les données. A ceci s'ajoute le manque des moyens qui caractérisent le personnel judiciaire œuvrant dans les milieux ruraux. Lorsque le Ministère Public décide d'exercer l'action publique, il communique les pièces au juge compétent pour en connaître. Par une requête aux fins de fixation d'audience, l'Officier du Ministère Public envoi le dossier au tribunal. Dans la pratique, il prend soin de libeller en fait comme en droit. La victime du viol d'enfant assistée ou représentée par ses parents ou tuteurs, peut aussi agir par voie d'une plainte ou d'une citation directe. Dans ce cas, elle aura la charge de la preuve puisqu'ayant forcé la main du Ministère Public.

\section{* LE TRIBUNAL COMPETENT EN MATIERE DE VIOL D'ENFANT.}

Il nous sera prématuré de fixer nos lecteurs sur le Tribunal compétent en cas de viol sans avoir dit en mot sur la peine

\section{- $\quad$ PEINE}

Les peines en matière de viol d'enfant sont prévues et punies par les articles 171 du code pénal livre II tel que modifié et complété par la loi n 06/018/ du 20 juillet 2006 et les articles 171 et 170 de la loi

\footnotetext{
${ }^{6}$ Article 2, Code de procédure pénale

${ }_{8}^{7}$ Article 192 de la loi n09/001 du 1er janvier 2009 portant protection de l'enfant.

8 Article 67 de la loi organique $n^{\circ} 13 / 011$-B du 11 Avril 2013 Portant organisation, fonctionnement et compétence des juridictions de l'ordre judiciaire en matière répressive
}

DOI: $10.9790 / 0837-2206023543 \quad$ www.iosrjournals.org $\quad 39 \mid$ Page


n'09/001 du 10 janvier 2009 portant protection de l'enfant.

L'article 171 du code pénal livre II tel que modifié et complété par la loi nº6/018 du 20 juillet 2006 dispose « si le viol ou l'attentat à la pudeur a causé la mort de la personne sur la quelle il a été commis, le coupable sera puni de la servitude pénale à perpétuité .»

L'article 170 de la loi nº9/001 du 10 janvier 2009 portant protection de l'enfant dispose que « le viol d'enfant est puni de sept à vingt ans de servitude pénale principale et d'une amande de huit cent mille à un million de francs congolais. Le minimum de la peine est doublé si le viol est le fait :

* Des ascendants de l'enfant sur lequel ou avec l'aide du quel le viol a été commis ;

* Des personnes qui ont autorité sur l'enfant ;

* De ses enseignants ou de ses serviteurs à gage ou les serviteurs des personnes ci-dessus ;

* des agents publics, des ministres de culte qui ont abusé de leur position pour le commettre, du personnel médical ou des assistants sociaux; des tradipraticiens envers les enfants confiés à leurs soins ;

* Des gardiens sur les enfants placés sous leur surveillance ; le minimum de la peine est également doublé ;

* S'il est commis avec l'aide d'une ou plusieurs personnes ;

* S'il est commis en public ;

- S'il a causé à la victime, une altération grave de la santé et/ou laisse de séquelles physiques et/ou psychologiques graves;

* S'il est commis sur un enfant vivant avec handicap ;

* S'il a été commis avec usage ou menace d'une arme.

Il sied de rappeler que si certaines circonstances font doublées la peine, en cas de viol d'enfant, seule la mort de la victime aggrave la peine car dans pareil cas, la peine à perpétuité est prévue. De ce qui précède, nous estimons que la loi n ${ }^{\circ} 09 / 001$ du 10 janvier 2009 ne réprime pas le viol ayant entraîné la mort de la victime. C'est ainsi que dans la pratique on doit se référer à l'article 171 du code pénal livre II; lequel du reste est dépassé en ce sens que certains actes jadis constitutifs d'attentat à la pudeur, constituent aujourd'hui le viol d'enfant étant donné que la conjonction sexuelle n'est plus la condition indispensable de l'infraction de viol d'enfant ;

Au regard des peines prévues en matière de viol d'enfant, le juge ne peut prononcer que les peines cumulée, c'est-a-dire la peines de servitude pénale et celle d'amende. Prononcer uniquement la peine de servitude pénale et ou celle d'amande constituerait une violation flagrantes de la loi. La peine étant lourde, le délinquant doit être examiné par un psychologue pour déterminer son état psychique afin d'établir sa responsabilité pénale.

\section{Le Tribunal compétant}

L'article 89 de la loi organique ${ }^{\circ} 13 / 011-\mathrm{B}$ du 11 Avril 2013 portant organisation, fonctionnement et compétences des juridiction de l'ordre judiciaire dispose que les tribunaux de Grande Instance connaissent des infractions punissables de la peine de mort et de celles punissables d'une peine excédent cinq ans de servitude pénale principale, hors le viol d'enfant est puni de sept à vingt ans de servitude pénale principale et d'une amande de huit cent mille à un million de francs congolais ou de la servitude pénale à perpétuité si la victime en est mort, il ne fait donc l'ombre d'aucun doute que le Tribunal compétent en matière de viol d'enfant est le Tribunal de Grande Instance.

\section{\&A PREUVE}

En matière pénale, la preuve est tout moyen permettant d'affirmer l'existence d'une infraction ou son absence, la culpabilité ou l'innocence du prévenu ${ }^{9}$.Lorsque le moyen est déterminé par la loi, la preuve est dite légale, dans les autres cas elle est dite libre. En cas des violences sexuelles l'Officier du Ministère Public ou le juge requiert d'office un médecin et un psychologue afin d'apprécier l'état de la victime de violence sexuelles et de déterminer les soins appropriés ainsi que évaluer l'importance du préjudice subi par celle-ci et son aggravation ultérieure ${ }^{10} \mathrm{Le}$ moyen de preuve peut consister en des simples indices : l'ensemble de faits connus à partir desquels on établit, au moyen de raisonnement indicatif, l'existence du fait contesté dont la preuve n'est pas directement possible. Ils sont aussi appelés présomptions judiciaires : faits dont on peut induire l'existence ou l'absence de l'infraction, la culpabilité ou l'innocence de la personne soupçonnée (empreintes, traces diverses, enregistrements magnétiques...)En matière pénale, faire la preuve c'est mettre à la disposition du juge les différents éléments pouvant déterminer la décision du juge. Dans un état de droit, d'aucun n'ignore que c'est dans le respect total de la loi et des droits fondamentaux de chacun que la preuve doit être administrée pour permettre au juge en toute quiétude d'avoir une vision des faits aussi fidele que possible de la réalité du

\footnotetext{
${ }^{9}$ PUNGWE NEMBA, guide pratique des magistrats du parquet, éd SDEMJ, Kin, 2006,p62.

${ }^{10}$ Article 14 bis, Loi n06/019 du 20 Juillet 2006,. 
cas qui lui est soumis ,c'est au juge en vertu du pouvoir discrétionnaire lui conféré par le loi d'apprécier et de voir quelle valeur à accorder à chacun des éléments de preuve qui lui sont soumis lors du procès. Deux questions peuvent être posées en matière de viol d'enfant, la première est celle de savoir qui a la charge de la preuve et la deuxième est la détermination de ce qui peut constituer un moyen de preuve.

\section{1). La charge de la preuve}

Dans un procès de viol d'enfant comme dans tout procès pénal, la charge de la preuve incombe au ministère public en sa qualité de l'organe poursuivant. Il doit apporter la preuve des faits reprochés au prévenu, leur caractère infractionnel ainsi que leur imputabilité au prévenu avant de requérir toute application de la peine ou mieux la condamnation du prévenu. En matière répressive comme en matière civile, le principe selon lequel la charge de la preuve incombe au demandeur est d'application ( actiri incombit probatio) c'est donc au Ministère Public entant qu'organe accusateur d'établir l'existence légale et matérielle de l'infraction et participation matérielle à celle -ci de la personne poursuivie, de même que sa participation morale. Dans le cas d'une citation directe, la charge de la preuve incombera à la partie citante et la tache du Ministère Public. Faudra-t-il le dire, sera d'acculer la partie citante aux fins que celui -ci apporte au tribunal la preuve qui peut l'aider à soutenir l'accusation. Dans un procès de viol d'enfant, l'élément déclencheur étant l'âge de la victime ; il appartiendra au Ministère Public et/ou à la partie citante d'apporter au juge la preuve de la minorité de la victime. C'est dans cette logique qu'avant de saisir le juge, le Ministère Public ou la partie citante doit se rassurer qu'il possède la preuve de la minorité de la victime. Certains principes qui caractérisent la procédure en République Démocratique du Congo sont à déplorer, ceux de croire que l'infraction de viol d'enfant étant trop grave pour ne pas dire importante, qu'il faut exiger à la personne poursuivie d'apporter la preuve de sa culpabilité, notamment celle de la minorité de la victime. Quelle justice à deux vitesses! Dans la pratique chaque fois que le Ministère Public accule la partie victime en lui demandant de lui apporter la preuve de sa minorité, celle-ci se fâche, craque la porte et accuse le ministère public de partial; Le Ministère Public aussi comme pour plaire à sa hiérarchie ou par ignorance fixe le dossier sans la preuve préalable de la minorité de la victime espérant que le juge pénal, étant actif, pourra couvrir ces vices. Ne dit-on pas que la preuve est l'âme du procès !

Si en matière pénale la preuve est libre, mais quand il s'agit de prouver l'âge de quelqu'un ou de la victime, la preuve n'est plus libre car le législateur exige que l'âge soit prouvé par un acte de l'état civil.

\section{Les actes de l'état civil}

Il faut entendre par acte de l'état civil un écrit établi par un officier de l'état civil et constatant un des événements affectant l'existence ou l'identification juridique d'une personne. II peut s'agir d'un acte de naissance, de mariage ou décès. Ou encore les actes authentiques qui constatent les principaux faits ou actes juridiques relatifs à l'état d'une personne. Dans le cas sous examen l'acte qui nous intéresse est l'acte de naissance parce qu'étant le premier acte de l'état civil d'une personne auquel le législateur accorde beaucoup de soins. Il ressort de l'analyse de l'article 76 du code de la famille que les fonctions d'officier de l'état civil sont remplies soit par les commissaire de zone rurale(Administrateur de Territoire) ;ou zone urbaine( Bourgmestre) ou sous sa direction par les agents subalternes qu'il désigne, soit par le chef de collectivité (chef de secteur) ou sous sa direction les agents subalternes qu'il désigne. Par rapport à la valeur que le législateur accorde à l'acte de naissance, celui-ci demeure un moyen sûr qui permet d'identifier et de prouver la qualité d'une personne.

De ce qui précède, ne constituent pas des actes de l'état civil au regard de la loi, la carte d'identité ou l'attestation de composition familiale établie par le bourgmestre. Toute naissance survenue sur le territoire de la République doit être déclarée à l'officier de l'état civil de la résidence du père ou de la mère dans les quatre vingt dix jours qui suivent la naissance. Notons que cette disposition est confirmée par l'article 16 de la loi nº9/001 du 10 janvier 2009 portant protection de l'enfant qui fixe le délai de la déclaration de la naissance à 90 jours. Passé ce délai, l'acte de l'état civil n'a que la valeur probante de simples renseignements toute fois; il en sera autrement s'ils sont inscrits au registre en vertu d'un jugement déclaratif ou supplétif tel que stipulé par l'article 98 du code de la famille. Aux termes de l'article 119 du code de la famille; il est fait obligation par le législateur aux 
hôpitaux, maternités ou formations médicales public ou privés de tenir un registre spécial sur lequel sont immédiatement inscrites, par ordre de date, les naissances qui y surviennent. Si dans les milieux ruraux de la République Démocratique du Congo certains organes de l'Etat peuvent faire défaut, ce n'est pas le cas des autorités faisant fonction de l'officier de l'état civil qui sont toujours présentes dans ces milieux. Au regard de ce qui vient d'être dit, pourquoi alors chaque fois qu'il ya viol d'enfant les victimes ne sont pas à mesure d'apporter la preuve de leur minorité ? Deux raisons seraient à la base. La première c'est l'ignorance de la population qui n'estime pas à juste valeur l'importance de l'acte de naissance ; la deuxième raison c'est la soif d'argent, qui caractérise certaines personnes de mauvaise foi qui font passer leurs filles majeures pour des mineures. Sciemment, elles cachent toutes les preuves justes pour obtenir du tribunal la condamnation des innocents.

L'article 14 bis de la loi nº6/019 du 20juillet 2006 modifiant et complétant le décret du 06 Août 1959 portant code de procédure pénale congolais stipule que conformément aux articles 48 et 49 ci-dessous, l'Officier du Ministère Public ou le juge requiert d'office un médecin et un psychologue, afin d'apprécier l'état de la victime des violences sexuelles et de déterminer les soins appropriés ainsi que d'évaluer l'importance du préjudice subit par celle-ci ou son aggravation ultérieure. Si dans la pratique, on requiert le médecin, généralement le psychologue est oublié. Et la conséquence en est que la victime ne sera plus mise en charge convenablement et pourra par la suite développer la disfonctionnement sexuel dû à la coercition sexuelle.

L'article 167 al 2 de la loi no 06/018 du 20 juillet 2006 modifiant et complétant le décret du 30 janvier 1940 portant code pénal congolais dispose que « tout attentat à la pudeur commis sans violences, ruse, ou menaces sur la personne ou à l'aide de la personne d'un enfant âgé de moins de dix-huit ans sera puni d'une servitude pénale de six mois à cinq ans. L'âge de l'enfant pourra être déterminé par un examen médical, à défaut d'état civil. »Dans la pratique le Ministère Public et le juge se fondent sur ces dispositions, pour soumettre la victime de viol d'enfant à des examens médicaux afin que le médecin dans un rapport médical leur détermine l'âge de la victime. Et très souvent le médecin estime en leur disant que l'âge de la fille varie entre tel et tel âge. Nous estimons que ceci est une lecture erronée et même une violation flagrante du droit de la défense car, on ne peut pas sur base d'une présomption médicale condamner une personne poursuivie. Aussi la loi sus évoquée qui renvoie à l'expertise médicale pour déterminer l'âge de l'enfant, à défaut d'acte d'état civil, traite de l'attentat à la pudeur et non du viol. Ne dit-on pas que La loi pénale est d'une interprétation stricte !

Il est plus raisonnable et même légal qu'en cas de viol d'enfant, que l'âge de la victime soit prouvé par une pièce d'état civil ou à défaut, par un certificat médical.

Dans un procès de viol d'enfant, l'âge de la victime n'est pas l'unique élément à prouver, il faut aussi apporter la preuve matérielle de l'infraction et le magistrat n'étant pas savant dans tous les domaines il lui est parfois recommandé de faire appel à des experts, notamment au médecin et les psychologue pour être élucidé.

\section{2). Le rapport médical.}

En matière pénale la preuve est libre. Cette liberté de preuve doit se faire dans le strict respect de la loi, de la dignité et des droits de la défense et ce, dans le seul but d'extirper le doute qui pourrait exister en faveur de la personne poursuivie et d'établir avec certitude l'existence de tous les éléments constitutifs de l'infraction. Dance cette démarche le ministère public n'agit pas seul, il est simplement l'acteur principal car d'autres acteurs dont le rôle n'est pas à négliger pour une bonne administration de la justice existent c'est notamment, l'interprète, le traducteur, le médecin, le psychologue. En cas de doute sur les renseignements recueillis, le magistrat instructeur peut requérir un expert ce, sur pied l'article 48 du code de procédure pénale. C'est dans cette logique que l'article 14 bis de la loi no $06 / 019$ du 20 juillet 2006 modifiant et complétant le décret du 06 août 1959 portant code de procédure pénale congolaise stipule que conformément aux articles 48 et 49 ci-dessus, l'Officier du Ministère Public ou le juge requiert d'office un médecin et un psychologue, afin d'apprécier l'état de la victime des violences sexuelles et déterminer les soins appropriés ainsi que d'évaluer l'importance du préjudice subi par celle -ci et son aggravation ultérieure. Pour ce qui est de la répression du viol d'enfant, le médecin et le psychologue jouent un rôle non moins négligeable, ils interviennent pour fixer le magistrat instructeur sur la santé physique et mentale de la victime ainsi que celle de la personne poursuivie.

Dans la plus part de cas chaque fois qu'il ya viol d'enfant c'est le personnel médical particulièrement le médecin, qui entre le premier en contact avec la victime pour les soins appropriés. Cette phase est à n'en point douter la plus importante pour la collecte des données pouvant conduire par la suite à établir ou infirmer tout lien entre la victime et la personne poursuivie d'une part et d'autre part entre la victime, les objets recueilles ainsi que les lieux des faits 
Notons cependant que l'article 14 bis de la loi sus évoquée n'autorise pas au médecin entant qu'expert de déterminer l'âge de la victime, à défaut d'acte de l'état civil. Et toute interprétation dans ce sens est erronée.

\section{CONCLUSION ET SUGGESTIONS}

L'enfant étant l'avenir de toute une nation, il nécessite l'attention particulière de la communauté internationale. Voilà pourquoi dans le but de permettre son épanouissement, le monde entier est mobilisé pour réprimer toute infraction dont l'enfant serait victime notamment le viol d'enfant. Lorsque le tribunal est saisi pour une question de viol d'enfant, l'une des préoccupations majeures du juge après avoir vidé la question relative à la procédure, est celle d'analyser la véracité des éléments de preuve à sa disposition. Cette analyse peut aboutir à la condamnation ou à l'acquittement du prévenu. L'important dans un procès de viol d'enfant c'est de prouver en amont la minorité de la victime et en aval les faits mêmes se rapportant au viol ainsi que la responsabilité du prévenu. Dans cette démarche, les actes de l'état civil en particulier l'acte de naissance, et le rapport médical et celui du psychologue sont d'une importance capitale. Nous souhaiterions que le législateur qui réprime le viol d'enfant puisse intégrer expressément dans une disposition que « l'âge de la victime pourra être déterminé par examen médical, à défaut d'acte de l'état civil. » étant donné que la loi portant protection de l'enfant ne le mentionne pas. Que les médecins et psychologues soient recrutés et affectés dans des milieux ruraux pour permettre à la population de se faire consulter par eux dans des hôpitaux, centres de santé et cabinet des psychologues qui sont aussi à pourvoir. Que chaque fois qu'il y a viol, que la victime soit traité non seulement par un médecin, mais aussi par un psychologue. La loi relative aux actes de l'état civil spécialement à l'acte de naissance n'est pas connue du grand public surtout dans les milieux ruraux. Voilà pourquoi il serait mieux aux autorités publiques et leurs partenaires de sensibiliser l'ensemble de la population sur la pertinence de l'acte de naissance surtout en cas de viol. Chaque parent doit être informé que la loi lui fait obligation de déclarer la naissance dans un délai de 90 jours qui suivent la naissance et sans frais. Le viol étant une infraction honteuse de par nos coutumes, les victimes préfèrent se taire ou trouver des solutions à l'amiable, lesquelles n'aboutissent souvent pas. Et lorsqu'elles se décident de saisir la justice, elles ont du mal à reconstituer et à produire les éléments de preuve. C'est pourquoi les victimes de viol ne peuvent pas attendre ni chercher à transiger, elles doivent directement porter l'affaire devant la justice et directement consulter le médecin et les non psychologues pour des soins appropriés, la détermination des conséquences par rapport à la santé et la collecte des données pouvant servir de preuve au moment opportun. 